\title{
Modelos mentais para representação atômica numa perspectiva de aprendizagem significativa
}

Mental models for atomic representation in a significant learning perspective

\author{
A. R. Pires*; A. L. F. Novais; L. M. Gomes; F. C. L. Ferreira \\ Programa de Pós-Graduação em Ensino de Física, Universidade Federal do Sul e Sudeste do Pará, 68500-000, \\ Marabá - PA, Brasil \\ *cientistadriano@yahoo.com.br
}

(Recebido em 08 de março de 2019; aceito em 13 de maio de 2019)

\begin{abstract}
Em diversas áreas da ciência é comum utilizar o termo modelo como uma representação analógica de um fenômeno, relacionando-o as memórias cotidianas para obter um quadro mental que possibilite a sua compreensão. Ao utilizar esse termo para definir fenômenos atômicos é necessário um amplo grau de abstração, pois o mesmo se distancia do cotidiano exigindo representações análogas a realidade. A partir da compreensão de que o termo modelo é em muitas áreas utilizado como uma representação abstrata é que este trabalho torna-se imprescindível. Outra importante contribuição é analisar a didática utilizada pelos professores de Ciências ao abordarem esse termo em suas práticas. Além do processo de formação dos modelos mentais na perspectiva da construção de conhecimento. O presente trabalho analisou um questionário contendo 3 (três) questões referentes a, por exemplo, constituição e movimento atômico. As análises realizadas após a aplicação do questionário revelaram dificuldades relacionadas ao processo em que ocorre o ensino e aprendizagem acerca do conteúdo referente aos modelos atômicos e nas representações e estruturas cognitivas utilizadas.

Palavras-chave: modelos mentais, modelos atômicos, aprendizagem significativa.
\end{abstract}

In some areas of science, the use of the term model as an analogical representation of a phenomenon, relating them as everyday memories to obtain a mental picture that enables their understanding. The use of the term for complete atomic facts is required by the wide angle of abstraction, by the same distance from every day requiring representatives from reality. The editing of the understanding of the model in many areas used as a statement is made in this work becomes essential. Other greater contribution is given to didactic apparatus for students of science to practice that term in their practices. In addition, the modeling process is in the perspective of knowledge building. The present study was designed to demonstrate three ways of identifying and participating actively. The analytics for the application in the issue related to the models in the participation in the participation in the teaching and teaching about the matter in the atomic in the networks and representational networks are cognitive used.

Keywords: atomic models, mental models, meaningful learning.

\section{INTRODUÇÃO}

A história evidencia que a evolução dos seres humanos esteve condicionada a utilização de modelos seja para representar, explicar e/ou mesmo entender a natureza e seus fenômenos. A explicação dos fenômenos da natureza a partir do conhecimento estruturado é responsável pela construção de um modelo científico. Nesse contexto, e utilizando os parâmetros filosóficos e científicos é que os modelos atômicos são fundamentados. A partir da concepção de que é uma tarefa árdua transmitir para o estudante o conceito que envolve o modelo científico é que tornase indispensável à utilização das Tecnologias da Informação e Comunicação (TIC) como recurso didático para estudar os conceitos que envolvem os modelos atômicos, pois de acordo com Vallin (1998) [1] as TIC são consideradas ferramentas que contribuem para o avanço na educação, pois possuem materiais dinâmicos em programas e softwares que atraem a atenção do estudantes provocando a interatividade e participação do estudante. 
Dall'asta (2004, p.17) [2] descreve os softwares como importantes ferramentas educacionais, estes: “[...] podem incorporar textos, sons, imagens, cores, mecanismos de percepção, fazendo-o aprender uma tarefa divertida na qual podem ser explorados os estímulos visuais e auditivos, movimentos, conteúdos das disciplinas escolares". Os software, os aplicativos e o datashow funcionam como ferramentas que possibilitam imagens mais próximas daquilo que se quer demonstrar, permitindo o desenvolvimento de representações indispensáveis a compreensão do assunto. Nesse sentido, Pozo e Crespo (2009) [3] fomentam que,

[...] Se as imagens que os estudantes percebem do mundo não são suficientes para compreender a estrutura da matéria, o ensino não consegue proporcionar sistemas de representação alternativos que permitam aos estudantes compreender a natureza. Os sistemas proposicionais que lhes são oferecidos matemáticos, algébricos, ou por meio de símbolos químicos e, apenas em alguns casos, analógicos - não seriam suficientes (POZO e CRESPO, 2009, p. 146) [3].

É necessária, portanto, a substituição da forma como o estudante representa a estrutura da matéria e de suas partículas constituintes, por uma proposta alternativa de linguagem, na qual os indícios perceptivos sejam abandonados gradativamente.

\section{MODELOS MENTAIS}

Os conceitos relativos aos Modelos Atômicos, a Física Nuclear entre outros, exigem um amplo grau de abstração, pois se distanciam do cotidiano. Para Jhonson-Laird (1983) [4] cada modelo mental representa uma possibilidade, ou seja, a maneira de avaliar a consistência de um conjunto de premissa é buscar um modelo no qual todas as afirmações se mantenham. Nesse sentido, Moreira (1999) [5], descreve o termo modelos mentais da seguinte maneira:

Modelos mentais são representações analógicas, um tanto quanto abstraídas, de conceitos, objetos ou eventos que dependem das impressões sensoriais, mas que podem ser vistos de qualquer ângulo (Imagens) e que em geral, não retêm aspectos distintos de uma dada instância de um objeto ou evento (MOREIRA,1999, p.184) [5].

Os seres humanos raciocinam naturalmente por meio de modelos mentais, porém, não sabem. Na sala de aula, por exemplo, constantemente, são formados modelos mentais por estudantes e professores de forma inconsciente. Para que os mesmos possam construir modelos coerentes cientificamente é necessário o acréscimo de informações, em função disso, forma-se um sistema cognitivo que capacita o sujeito a compreensão do que estar sendo estudado. A teoria de JhonsonLaird (1983) [4] sugere que:

As pessoas raciocinam com modelos mentais, os quais seriam como blocos de construção cognitivos que podem ser combinados e recombinados conforme necessário. A compreensão significativa de um conceito, evento ou objeto implica a construção de um modelo mental de trabalho deste conceito, evento ou objeto (MOREIRA e KREY, 2006, p. 357) [6].

Nesse sentido é importante verificar como são as imagens mentais dos estudantes acerca de um determinado conceito, já que a partir delas, podemos compreender os modelos mentais préexistentes e assim verificar a necessidade de inferências científicas significativas, afim de construir novos modelos, como é salientado no trecho a seguir:

Tais modelos iniciais podem vir a ser depurados com o tempo, em maior ou menor grau dependendo do envolvimento e interesse do sujeito por mais conhecimentos naquele domínio. Por exemplo, na escola, o conhecimento assimilado interage com os modelos existentes para produzir novos modelos. Estes apresentam um sistema mais rico e empregam propriedades novas para descrever e explicar eventos no domínio em questão. Para que o sujeito venha a adquirir modelos mentais próximos dos modelos científicos é necessário bastante tempo e esforço de sua parte (BORGES, 1998, p. 25) [7]. 
As analogias são mecanismos usados para estabelecer comparações que dependem da percepção e imaginação, as quais resultam em imagens e modelos mentais, estruturas cognitivas fundamentais ao processo de aprendizagem. Dessa forma, torna-se evidente que a utilização dos modelos mentais como canal alternativo de linguagem para a representação atômica, possibilita um avanço significativo nesse processo. Moreira (1999) [5] ressalta que

\begin{abstract}
A percepção é fonte primária de modelos mentais do mundo. Contudo, seres humanos podem, evidentemente, construir modelos mentais também por atos de imaginação. Isso significa que as proposições podem também ser referidas a mundos hipotéticos ou imaginários. Reside aí uma suposição básica da teoria de Laid: representações proposicionais são interpretadas em relação a modelos mentais (1983, p.156). Isso quer dizer que, para ele, a semântica, i.e., a parte do significado, da linguagem mental, remete representações proposicionais a modelos mentais de mundos reais ou imaginários (MOREIRA, 1999, p. 187) [5].
\end{abstract}

O objetivo do presente trabalho é investigar e analizar os modelos mentais construídos por alunos sobre estruturas atômicas visando à aprendizagem significativa.

\title{
3. MATERIAL E MÉTODOS
}

O presente trabalho foi desenvolvido a partir do método quanti-qualitativo. Para Chizzotti (2005) [8], "A pesquisa quanti-qualitativa, pressupõe que o pesquisador descreve, explica, participa, compreende e interpreta". O material utilizado para obter os resultados foi um questionário contendo 3 (três) questões de caráter investigativo. O referido questionário foi aplicado para 20 estudantes do $8^{\circ}$ ano do Ensino Fundamental na Escola Municipal Lucíolo Oliveira Rabelo, situada no município de Goianésia do Pará - PA. Para a coleta de dados, aplicouse o Termo de Assentimento Livre e Esclarecido (TCLE) com os envolvidos na pesquisa. As questões eram referentes ao "átomo" e foram aplicadas com o objetivo de identificar possíveis problemas no decorrer do processo de ensino e aprendizagem referente conceitos que envolvem os modelos atômicos. A Tabela 1 apresenta o questionário aplicado aos alunos.

Tabela 1: Questões do questionário aplicado aos alunos.

\begin{tabular}{|c|c|}
\hline Questão & Descrição das questões \\
\hline 1 & $\begin{array}{l}\text { De acordo com seus conhecimentos o átomo é maciço (compacto, sem parte oca) } \\
\text { ou possui um espaço vazio ou desconhecem? }\end{array}$ \\
\hline 2 & $\begin{array}{l}\text { De acordo com seus conhecimentos o átomo é uma partícula estática ou está em } \\
\text { movimento ou desconhecem? }\end{array}$ \\
\hline 3 & $\begin{array}{l}\text { De acordo com seus conhecimentos o modelo atômico proposto por Dalton, } \\
\text { Thomson, Rutherford e Quântico, o que representam didaticamente? a) uma esfera } \\
\text { maciça, indivisível e homogênea b) uma esfera maciça compacta de carga positiva } \\
\text { imersa por cargas negativas c) a um núcleo positivo concentrando praticamente } \\
\text { toda massa, orbitado por elétrons d) a um núcleo de cargas positiva envolvido por } \\
\text { uma núvem eletrônica onde há uma probalidade de se concentra elétrons e) } \\
\text { desconheço proposto átomo é uma partícula estática ou está em movimento ou } \\
\text { desconhecem. }\end{array}$ \\
\hline
\end{tabular}

\section{RESULTADOS E DISCUSSÃO}

Os resultados obtidos a partir da aplicação do questionário sobre a constituição atômica mostraram que os estudantes entrevistados já conheciam o assunto. No entanto, $60 \%$ dos estudantes entrevistados identificaram o átomo como um objeto maciço (compacto, sem partes ocas), $30 \%$ dos entrevistados relataram que o átomo possui espaço vazios e $10 \%$ deles não tinha 
conhecimento sobre o assunto. A Figura 1 apresenta o percentual de estudantes que participaram da entrevista relacionada a constituição atômica.

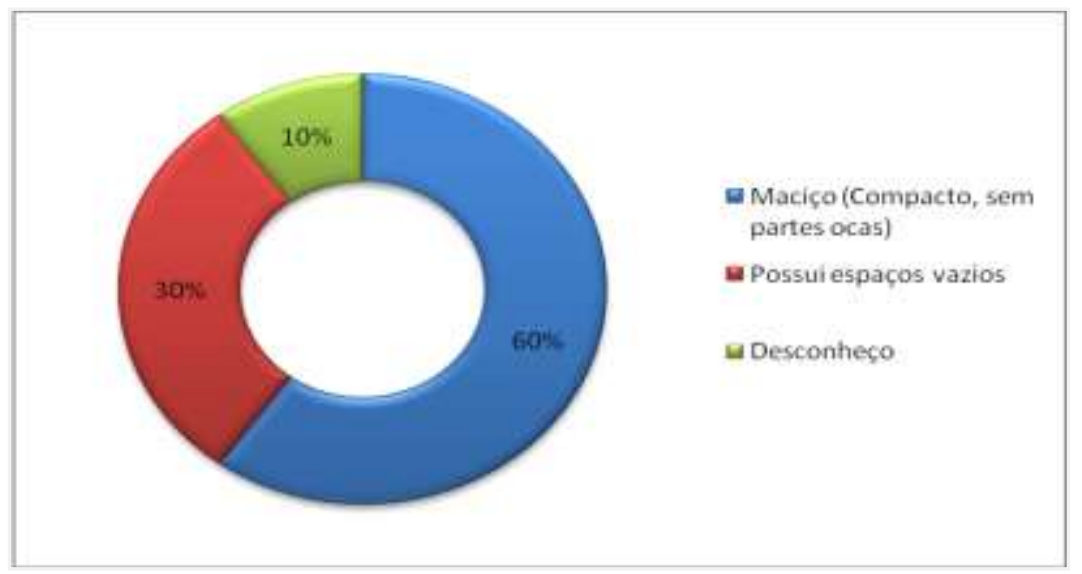

Figura 1: Percentual de estudantes que foram entrevistados sobre a constituição do átomo.

A percepção dos alunos que foram entrevistados sobre o conhecimento adquirido com o estudo apresenta a noção macroscópica proveniente dos sentidos, nesse caso visão e tato, reforçando a ideia de um átomo compacto. Pozo e Crespo (2009) [3] questionam a resistência dos conhecimentos prévios em modificar-se como consequência da instrução,

Se a instrução praticamente não afeta as concepções sobre a continuidade ou descontinuidade da matéria, a ativação dessas concepções, em compensação, é muito influenciada pela aparência material das substâncias apresentadas. A ideia de vazio é utilizada com mais freqüência para representar a matéria em estado gasoso e, em muito menor medida, para os sólidos. Os líquidos, por sua vez, ficam em um nível de dificuldade intermediário (POZO, CRESPO e SANZ, 1993 apud POZO e CRESPO, 2009, p.154) [3].

Da mesma forma os entrevistados que alegam conhecer a partícula fundamental da matéria ficaram divididos entre o que vêem e o que estudaram acerca de seu movimento e o de suas partículas elementares. A Figura 2 apresenta o percentual de estudantes que possuem ou não conhecimento sobre o movimento dos átomos.

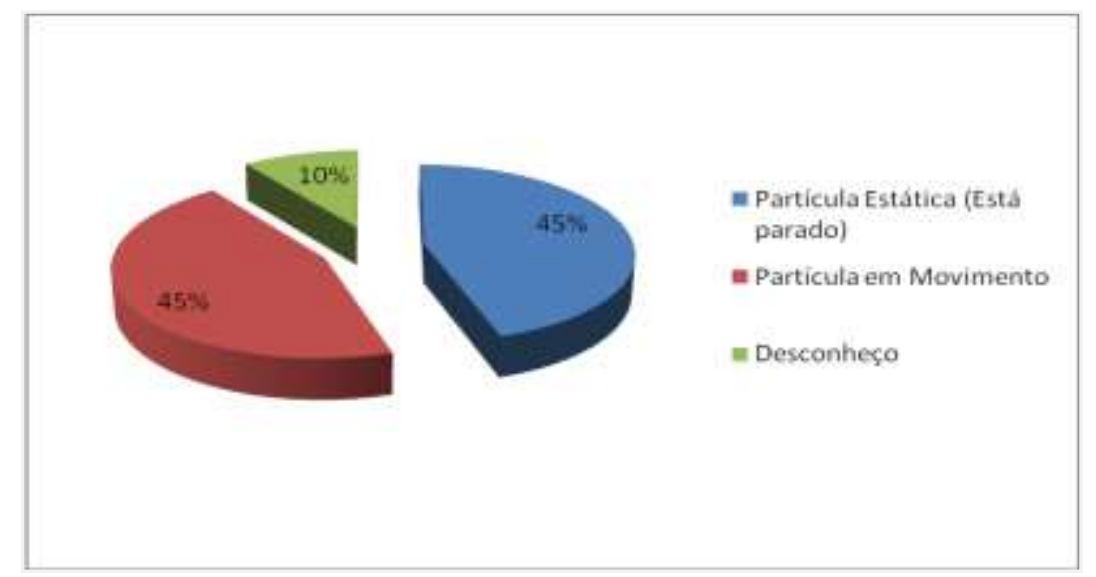

Figura 2: Conhecimento dos estudantes sobre o movimento do átomo.

Em azul, observa-se que $45 \%$ dos estudantes entrevistados acreditam que o átomo é uma partícula estática (está parado). Em vermelho, observa-se que $45 \%$ dos estudantes entrevistados acreditam que o átomo é uma partícula em movimento. Em verde, observa-se que $10 \%$ dos estudantes entrevistados desconhecem como o átomo se movimenta (Figura 2). 
A Figura 2 mostra o grau de conhecimento dos estudantes sobre o movimento do átomo, percebe-se que a amplia ainda mais a problemática decorrente da dificuldade que os estudantes têm em abandonar os indícios perceptivos como fonte de representação da matéria, sendo que $45 \%$ dos entrevistados compreendem o átomo como uma partícula inerte. Pozo e Crespo (2009) [3] apontam que,

De acordo com o modelo de mudança conceitual, esta representação diferenciada do movimento da matéria em seus diferentes estados de agregação poderia ser devida ao fato de os estudantes não diferenciarem entre o movimento intrínseco das partículas que compõem um material e o movimento aparente desse mesmo material, ou seja, sua aparência perceptiva. Essa falta de diferenciação entre o nível microscópico de análise da matéria, que é o que a química proporciona, e nossa percepção macroscópica dessa mesma matéria faz com que eles atribuam movimento intrínseco aos gases, mas não aos sólidos; ao mesmo tempo, no caso dos líquidos, eles tendem a atribuir movimento quando o liquido tem um movimento aparente (por exemplo, no mar ou em um refrigerante com gás) e, em compensação, fazem um interpretação estática quando o líquido não se movimenta, com isso, a variável relevante seria não tanto o estado de agregação quanto seu estado aparente de movimento ou repouso (POZO e CRESPO, 2009, p.152) [3].

A Figura 3 mostra os resultados obtidos na questão 3 que trata do modelo atômico proposto por Dalton, Thomson, Rutherford e Quântico que representa didaticamente: a) uma esfera maciça, indivisível e homogênea b) uma esfera maciça compacta de carga positiva imersa por cargas negativas c) a um núcleo positivo concentrando praticamente toda massa, orbitado por elétrons d) a um núcleo de cargas positiva envolvido por uma nuvem eletrônica onde há uma probalidade de se concentra elétrons e) desconheço.

Considerando-se as propostas de representação didática para o átomo, adotadas pelo professor, a evolução nos modelos atômicos exige dos estudantes um aumento no grau de imaginação e abstração, na perspectiva da construção de informações que possibilite a aprendizagem. Dessa forma, identificou-se que tal exigência influencia significativamente no processo de formação de imagens e como consequência nos modelos mentais. A Figura 3 apresenta o percentual de acertos dos estudantes em relação ao modelo atômico proposto por Dalton, Thomson, Rutherford e Quântico representado didaticamente.

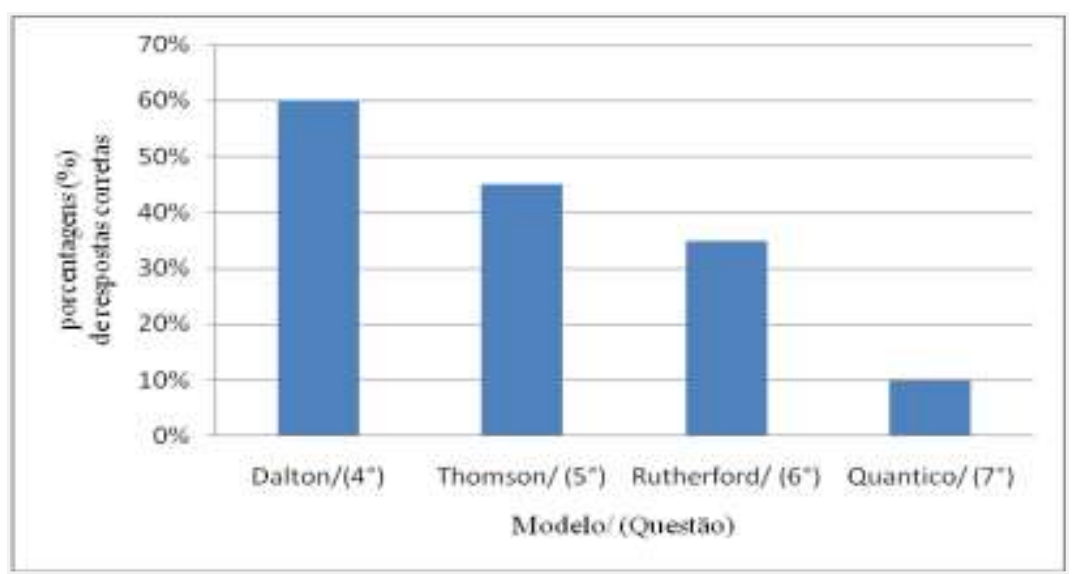

Figura 3: Porcentagem das respostas corretas para a questão 3 sobre os modelos atômicos aplicados no questionário.

Nesse sentido, fica evidente a necessidade urgente da reflexão nos aspectos didático e cognitivo, haja vista o decréscimo nos acertos observados $60 \%$ até $10 \%$. Sem contar a própria ineficiência dos recursos adotados pelo professor, que ainda preconiza a utilização exclusiva de quadro e pincel, informação dada pelos os estudantes entrevistados. 
Segundo Moreira (2006, p. 19) [9], “[...] uma das condições para ocorrência de aprendizagem significativa é que o material a ser aprendido seja relacionável (ou incorporável) à estrutura cognitiva do aprendiz, de maneira não arbitrária e não literal. Um material com estas características é dito como potencialmente significativo."

\section{CONCLUSÃO}

O presente trabalho apresentou as dificuldades encontradas por professores no processo ensino e aprendizagem no que se refere a transmissão do termo modelo. Enfatizou também que é necessário um amplo grau de abstração para compreender os conceitos que envolvem os modelos atômicos. No decorrer da investigação foi observado que a aprendizagem significativa ocorre quando novas ideias ou conceitos ancoram-se a conceitos relevantes já existentes na estrutura cognitiva do indivíduo. Portanto, é necessário adotar metodologias que garantam aos estudantes mecanismos essenciais a aprendizagem.

Os resultados obtidos a partir da aplicação do questionário a respeito da constituição atômica, mostrou que os estudantes entrevistados já conheciam o assunto. No entanto, $60 \%$ dos estudantes entrevistados identificaram o átomo como um objeto maciço (compacto, sem partes ocas), $30 \%$ dos entrevistados relataram que o átomo possui espaço vazios e $10 \%$ dos estudantes não tinham conhecimento sobre o assunto. Os resultados obtidos a partir da aplicação do questionário a respeito do conhecimento dos estudantes acerca do movimento atômico mostrou que $45 \%$ dos estudantes entrevistados acreditam que o átomo é uma partícula estática (está parado), 45\% dos estudantes entrevistados acreditam que o átomo é uma partícula em movimento e $10 \%$ dos estudantes entrevistados desconhecem como o átomo se movimenta. Outro importante resultado obtido é que o uso das TIC's como também experimentos utilizados de maneira correta, nas salas de aula, proporcionam metodologias inovadoras e diversificadas, as quais geram motivação dos estudantes pelas aulas e conteúdos. Dessa forma, tais recursos viabilizam a quebra de barreiras macroscópicas sensoriais garantindo a compreensão da evolução de representação do átomo a partir de modelos mentais potencializadas e cientificamente coerentes enriquecendo o processo de ensino e aprendizagem.

\section{AGRADECIMENTO}

Os autores agradecem à CAPES, ao CNPq e ao MNPEF pelo apoio nas pesquisas.

\section{REFERÊNCIAS BIBLIOGRÁFICAS}

1. Vallin C. Como usar o computador na escola. São Paulo: Editora Moderna; 1998.

2. Dall’asta RJ. A transposição didática no Software Educacional. Passo Fundo: UPF; 2004.

3. Pozo JI, Crespo MAG. A aprendizagem e o ensino de ciências: do conhecimento cotidiano ao conhecimento científico.Porto Alegre: Artmed; 2009.

4. Johnson-Laird PN. Mental Models. Cambridge, MA: Harvard University Press; 1983.

5. Moreira MA. Teorias de aprendizagem. São Paulo: EPU; 1999.

6. Moreia MA, Krey I. Dificuldades dos alunos na aprendizagem da lei de Gauss em nível de física geral à luz da teoria dos modelos mentais de Johnson-Laird. Rev Bras Ens Fís. 2006;28(3):353-360.

7. Borges AT. Modelos mentais de eletromagnetismo. Cad Bras Ens Fís. 1998;15(1):7-31.

8. Chizzotti A. Pesquisa em Ciências humanas e Sociais. $7^{\circ}$ ed. São Paulo: Cortez, 2005.

9. Moreira MA. A teoria da aprendizagem significativa e sua implementação em sala de aula. Brasília: Universidade de Brasília; 2006, 186 p. 